\title{
Effects of various etching protocols on the flexural properties and surface topography of fiber-reinforced composite dental posts
}

\author{
Juthatip AKSORNMUANG, Chanya CHUENARROM and Natjira CHITTITHAWORN \\ Department of Prosthetic Dentistry, Faculty of Dentistry, Prince of Songkla University, Hat Yai, Songkhla, Thailand \\ Corresponding author, Juthatip AKSORNMUANG; E-mail: juthatip.a@gmail.com
}

\begin{abstract}
The purpose of this study was to evaluate the flexural properties and surface topography of fiber posts surface-treated with various etching protocols. Seventy each of three types of fiber posts: RelyX Fiber Post, Tenax Fiber Trans, and D.T. Light-Post Illusion X-Ro, were randomly divided into 7 groups: no surface treatment, surface treated with hydrofluoric acid (HF) $4.5 \%$ for $60 \mathrm{~s}$, HF $4.5 \%$ for 120 s, HF $9.6 \%$ for $15 \mathrm{~s}$, HF $9.6 \%$ for $60 \mathrm{~s}$, HF $9.6 \%$ for $120 \mathrm{~s}$, and treated with $\mathrm{H}_{2} \mathrm{O}_{2} 24 \%$ for 10 min. The specimens were then subjected to a three-point bending test. Surface topographies of the posts were observed using a SEM. The results indicate that fiber post surface pretreatments had no adverse effects on the flexural properties. However, the fiber posts treated with high HF concentrations or long etching times seemed to have more surface irregularities.
\end{abstract}

Keywords: Fiber posts, Post surface etching, Flexural properties, Surface topography

\section{INTRODUCTION}

Currently, fiber-reinforced composite posts, socalled fiber posts, are routinely utilized to restore endodontically treated teeth in dental clinical practice. Several studies have supported the benefits of fiber posts in reducing the incidence of root fracture, and in providing favorable or reparable fracture pattern $s^{1-3)}$. Moreover, using fiber posts provides superior aesthetics, easier removal, and shorter treatment visits, in comparison to cast metal posts and cores.

Even though the utilization of a fiber post could reduce the incidence of root fracture, failure can still occur through decementation of the fiber post from the canal ${ }^{4,55}$. The flexibility of fiber posts allows them to consensually bend with dentin during function, resulting in debonding of the interface when the bond strength is inadequate. Failure could occur either at the resin-dentin or the resin-post interface. A previous study reported that debonding between fiber posts and luting resin took place when the root canal dentin was efficiently bonded ${ }^{6}$. Non-treated fiber posts have a relatively smooth surface, which limits mechanical interlocking with resin cements, compared with serrated or threaded fiber posts. Post surface treatments have been recommended to improve adhesion by facilitating chemical and micromechanical interfacial interactions of the post and the luting resin. These procedures fall into three categories: mechanical treatment (e.g. air abrasion or sand blasting), chemical treatment with hydrofluoric acid or hydrogen peroxide at various concentrations and durations, and combinations of mechanical and chemical methods. One study revealed that post surface abrasion produced undesirable surface changes ${ }^{7}$. Chemical surface etching is a convenient way to increase surface roughness of the fiber post, and to subsequently increase the bond strength ${ }^{8-10}$.

Hydrofluoric acid (HF) etching is often employed to enhance the bond strength of silica-containing substrates through micromechanical retention. The active mechanism in HF etching is the dissolution of silicon dioxide, which subsequently creates a rough surface, facilitating mechanical interlocking with the applied adhesive ${ }^{10)}$. Various HF concentrations and etching times have been proposed for fiber post etching protocols. Utilization of a low HF concentration, approximately $4-5 \%$, was suggested for $60-120$ s etching times ${ }^{11-13)}$. However, the high 9.6\% HF concentration has been normally used with shorter etching times in the range $15-60 \mathrm{~s}^{10,14)}$. One study demonstrated the highest bond strengths for posts etched with $9.6 \%$ HF for the comparatively long $120 \mathrm{~s}$ duration. The hypothesis that $\mathrm{HF}$ etching possibly damages integrity of the post and thereby reduces its strength has been suggested ${ }^{15)}$. However, such deterioration of properties would depend on the acid, its concentration, and etching time. Hydrogen peroxide etching has been promoted because it selectively dissolves the matrix without damage to the fibers ${ }^{16)}$. Monticelli et al. reported that etching fiber posts with $24 \% \mathrm{H}_{2} \mathrm{O}_{2}$ for 10 min could enhance their interfacial bonding strength to resin composites ${ }^{8)}$. The limits of a chemical surface treatment of fiber posts are determined by damage to the properties of the post. The purpose of the present study was to evaluate the flexural properties and surface topography of three types of fiber post, when surface treated with select etching protocols. The null hypothesis was that the alternative surface treatments do not affect the flexural properties of the posts.

\section{MATERIALS AND METHODS}

Seventy each of the following three types of fiber post: RelyX Fiber Post size \#2 (3M ESPE, St. Paul, MN, USA), Tenax Fiber Trans size TFT 15 (Coltène/Whaledent, Altstätten, Switzerland), and D.T. Light-Post Illusion 
Table 1 Materials used in the study

\begin{tabular}{|c|c|c|}
\hline Material & Manufacturer & Composition \\
\hline RelyX Fiber Post & $\begin{array}{l}\text { 3M ESPE, St. Paul, } \\
\text { MN, USA }\end{array}$ & $\begin{array}{l}\text { Glass fibers, epoxy-resin matrix containing } \\
\text { zirconia fillers }\end{array}$ \\
\hline Tenax Fiber Trans & $\begin{array}{l}\text { Coltène/Whaledent, } \\
\text { Altstätten, Switzerland }\end{array}$ & Glass fiber, methacrylate resin \\
\hline $\begin{array}{l}\text { D.T. Light-Post Illusion } \\
\text { X-RO }\end{array}$ & $\begin{array}{l}\text { RTD, St. Egrève, } \\
\text { France }\end{array}$ & $\begin{array}{l}\text { Quartz fibers, epoxy resin, catalyst, } \\
\text { colored pigment }\end{array}$ \\
\hline Porcelain Etch Gel & $\begin{array}{l}\text { Pulpdent, Watertown, } \\
\text { MA, USA }\end{array}$ & $\begin{array}{l}\text { Hydrofluoric acid } 9.6 \% \text {, ethyl alcohol, } \\
\text { gel base }\end{array}$ \\
\hline IPS Ceramic Etching Gel & $\begin{array}{l}\text { Ivoclar Vivadent, } \\
\text { Schaan, Liechtenstein }\end{array}$ & $\begin{array}{l}\text { Hydrofluoric acid } 4.5 \% \text {, water thickener, } \\
\text { pigment }\end{array}$ \\
\hline Hydrogen Peroxide Sol. & $\begin{array}{l}\text { Vidhyasom, Bangkok, } \\
\text { Thailand }\end{array}$ & Hydrogen Peroxide Sol. $35 \%$ diluted to $24 \%$ \\
\hline
\end{tabular}

X-Ro size \#1 (RTD, St. Egrève, France), were used in this study. Each type of fiber posts was randomly divided into 7 treatment groups, 10 posts per group, and the surface treatments were; Group 1 -no surface treatment (Control), Group 2 -surface treated with hydrofluoric acid (HF) at 4.5\%, IPS Ceramic Etching Gel (Ivoclar Vivadent, Schaan, Liechtenstein), for $60 \mathrm{~s}$ (HF4.5\%$60 \mathrm{~s})$, Group 3 -surface treated with $\mathrm{HF}$ at $4.5 \%$ for 120 s (HF4.5\%-120s), Group 4 -surface treated with $\mathrm{HF}$ at $9.6 \%$, Porcelain Etch Gel (Pulpdent, Watertown, MA, USA) for $15 \mathrm{~s}$ (HF9.6\%-15s), Group 5-surface treated with $\mathrm{HF}$ at $9.6 \%$ for $60 \mathrm{~s}$ (HF9.6\%-60s), Group 6 -surface treated with $\mathrm{HF}$ at $9.6 \%$ for $120 \mathrm{~s}$ (HF9.6\%120 s), Group 7 - surface treated with hydrogen peroxide $\left(\mathrm{H}_{2} \mathrm{O}_{2}\right)$ at $24 \%$ (Vidhyasom, LTD, Bangkok, Thailand) for $10 \mathrm{~min}\left(\mathrm{H}_{2} \mathrm{O}_{2} 24 \%-10 \mathrm{~min}\right)$. The materials used in this study are presented in Table 1 .

In the surface treatment of a post, the chemical agent was filled into a silicone pit, $20 \mathrm{~mm}$ in height and $3 \mathrm{~mm}$ in diameter. The post was entirely submerged into the surface treatment agent. After the surface etching, the post was rinsed with running water for $30 \mathrm{~s}$ and dried with air blow. The post was then placed on the individual post shape silicone mold that had a marking for the middle point along length of the post, and a marking was put on the post exactly at its middle. Each specimen was subjected to a three-point bending test in a universal testing machine (Lloyd instruments, LRX-Plus, AMETEK Lloyd Instrument, Hampshire, $\mathrm{UK})$ with a $250 \mathrm{~N}$ load cell. The crosshead was in the middle between two supports with $10 \mathrm{~mm}$ span, and the crosshead speed was set at $1 \mathrm{~mm} / \mathrm{min}$. The load and deflection were recorded by the control software for the universal testing machine (Nexygen, AMETEK Lloyd Instrument). The maximum load from the bending test was recorded for each post, as the main characteristic to be analyzed. Load-deflection ratio within elastic limit of each testing graph was calculated to signify the elastic property altering after surface treatments.

Surface topography of the treated fiber posts was observed using a scanning electron microscope (SEM, Quanta400, FEI, Hillsboro, OR, USA). The posts were handled with the same protocol preceding the threepoint bending test. After drying, each post was mounted on a brass tablet and gold sputter-coated. The surface topography was then observed and SEM micrographs were taken.

The data on maximum load and load-deflection ratio, its mean and standard deviation (SD), are reported and statistically analyzed. Since normality of data distribution and homogeneity of variance (Levene's test) were detected, the maximum loads and loaddeflection ratios were therefore compared between the 7 treatment groups using parametric one-way ANOVA at 95\% confidence level. In case significance was indicated, a further multiple comparison of means was performed using Tukey's HSD test.

\section{RESULTS}

Table 2 shows the mean and standard deviation of maximum load in Newtons (N), obtained from the threepoint bending test, for each type of fiber post and each surface treatment. For RelyX Fiber Post, the maximum load ranged from 96.90 to $114.68 \mathrm{~N}$. It was found that the surface treatment affected the flexural load of RelyX Fiber Post ( $p=0.003)$. Tukey's HSD multiple comparison test indicated a significant difference in the maximum load between the groups HF9.6\%-60s and $\mathrm{H}_{2} \mathrm{O}_{2} 24 \%$ $10 \mathrm{~min}(p=0.12)$, and between the groups HF9.6\%-120s and $\mathrm{H}_{2} \mathrm{O}_{2} 24 \%$-10min $(p=0.24)$. The maximum loads did not differ significantly between the other treatments of RelyX Fiber Post.

Table 3 displays the mean and standard deviation of the load-deflection ratios $(\mathrm{N} / \mathrm{mm})$ calculating from the testing graphs. There was no significant difference 
Table 2 Maximum load (Newton, Mean(SD) in) from three-point bending test of each type of fiber post after alternative surface treatments

\begin{tabular}{lccc}
\hline Surface treatment & RelyX Fiber Post & Tenax Fiber Trans & D.T. Light-Post \\
\hline Control & $111.48(8.69)$ & $107.11(7.10)$ & $76.54(2.07)$ \\
HF4.5\%-60s & $100.47(12.70)$ & $109.31(8.99)$ & $77.47(5.61)$ \\
HF4.5\%-120s & $110.15(15.59)$ & $108.61(7.95)$ & $76.46(4.95)$ \\
HF9.6\%-15s & $109.38(6.41)$ & $109.87(9.04)$ & $75.58(4.72)$ \\
HF9.6\%-60s & $114.68(9.22)^{* * \#}$ & $108.25(7.40)$ & $74.41(3.01)$ \\
HF9.6\%-120s & $113.44(4.64)^{*}$ & $111.75(9.57)$ & $75.65(6.49)$ \\
$\mathrm{H}_{2} \mathrm{O}_{2} 24 \%-10 \mathrm{~min}$ & $96.90(15.51)^{\#}$ & $111.78(7.14)$ & $76.00(3.19)$ \\
\hline
\end{tabular}

Values with the same superscript characters indicate significant differences $(p<0.05)$.

Table 3 Load-deflection ratio (N/mm, Mean(SD) within elastic limit from three-point bending test of each type of fiber post after alternative surface treatments

\begin{tabular}{lccc}
\hline Surface treatment & RelyX Fiber Post & Tenax Fiber Trans & D.T. Light-Post \\
\hline Control & $154.10(2.51)$ & $118.47(5.74)$ & $112.73(2.38)$ \\
HF4.5\%-60s & $140.46(5.70)$ & $115.63(6.64)$ & $111.30(5.04)$ \\
HF4.5\%-120s & $141.13(13.96)$ & $118.77(3.96)$ & $115.70(3.07)$ \\
HF9.6\%-15s & $145.10(6.00)$ & $119.18(3.76)$ & $114.30(4.15)$ \\
HF9.6\%-60s & $142.60(1.39)$ & $120.02(2.90)$ & $120.30(7.40)$ \\
HF9.6\%-120s & $143.10(7.96)$ & $124.10(3.03)$ & $119.38(2.97)$ \\
$\mathrm{H}_{2} \mathrm{O}_{2} 24 \%-10 \mathrm{~min}$ & $146.90(5.47)$ & $118.43(3.44)$ & $113.50(5.70)$ \\
\hline
\end{tabular}

No significant difference among surface treatment groups for all types of fiber posts.

in load-deflection ratios among 7 surface treatments for all types of tested posts $(p>0.05)$. The control group of RelyX Fiber Post appeared to have a higher loaddeflection ratio than those of the other groups. However, significant difference was not detected.

The results show maximum loads ranging from 107.11 to $111.78 \mathrm{~N}$ for Tenax Fiber Trans, and from 74.41 to $77.47 \mathrm{~N}$ for D.T. Light-Post. One-way ANOVA indicates that there are no significant differences in the maximum load between the 7 surface treatments, as the $p$-values are 0.839 and 0.845 , respectively. In other words, the alternative surface treatments did not affect the flexural strength of Tenax Fiber Trans and D.T. Light-Post.

SEM micrographs of RelyX Fiber post surfaces in the control and all experimental groups are shown in Fig. 1. In the control group, the post surface was mostly covered by the resin matrix, with a few fibers exposed on the outer surface (Fig. 1a). The post surfaces showed no obvious changes after treatments with HF4.5\%-60s, HF9.6\%-15s, HF9.6\%-60s, or $\mathrm{H}_{2} \mathrm{O}_{2} 24 \%-10$ min (Figs. 1b, d, e, and g). However, the glass fibers were etched into small pieces by the treatment with $\mathrm{HF}$ at $4.5 \%$ for $120 \mathrm{~s}$ (Fig. 2-arrows). Treatment with the strong acid $\mathrm{HF}$ at $9.6 \%$ for $120 \mathrm{~min}$ had dislodged all fibers from the surface, leaving only fiber imprinted grooves on the zirconia particle filled matrix (Fig. 2-asterisks).

For Tenax Fiber Trans (Fig. 3), the post surface in the control group was tightly covered by the resin matrix with only few fibers exposed on the outer surface (Fig. 3a). The surface appeared intact when treated with $\mathrm{HF} 9.6 \%-15 \mathrm{~s}$ or $\mathrm{H}_{2} \mathrm{O}_{2} 24 \%$-10min (Figs. 3a and g). Some glass fiber bundles were removed when the post was treated with HF4.5\%-60s (Fig. 3b). Glass fibers on the outer surface of a post were mostly wiped out when the post was immersed in $\mathrm{HF}$ at $4.5 \%$ for $120 \mathrm{~s}$, or in $\mathrm{HF}$ at $9.6 \%$ for 60 or $120 \mathrm{~s}$ (Figs. 3c, e, and f). Several micro-pores inside the resin matrix were observed in the groups that surface-treated with $\mathrm{HF}$ at 4.5 and $9.6 \%$ for $60 \mathrm{~s}$ or longer as shown by the asterisks in Fig. 4.

SEM micrographs of D.T. Light-Post surfaces are shown in Fig. 5. In the control group, the post surface was covered by the resin matrix with some fibers exposed on the outer surface (Fig. 5a). The surfaces treated with 


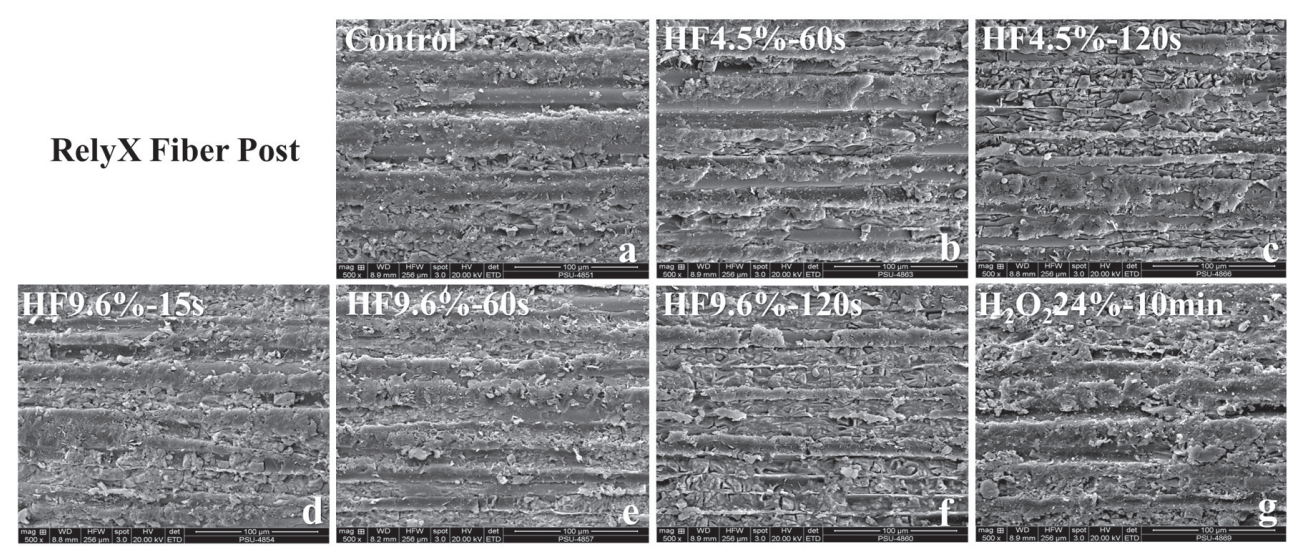

Fig. 1 SEM micrographs of RelyX Fiber Post surfaces before and after the various surface treatments.

In the control group, the post surface was mostly covered by the resin matrix, with a few fibers exposed on the outer surface (a). The post surface did not obviously change when treated with, HF4.5\%-60s, HF9.6\%-15s, HF9.6\%-60s, or $\mathrm{H}_{2} \mathrm{O}_{2} 24 \%$-10min (b, d, e, and g). However, the glass fibers were broken into small pieces by etching, when treated with $\mathrm{HF}$ at $4.5 \%$ for $120 \mathrm{~s} \mathrm{(c).} \mathrm{When} \mathrm{treated} \mathrm{with} \mathrm{strong} \mathrm{acid} \mathrm{HF} \mathrm{9.6 \%} \mathrm{for} 120 \mathrm{~min}$, all the etched fibers were dislodged from the surface, leaving fiber imprint lines on the zirconia-filled matrix (f).
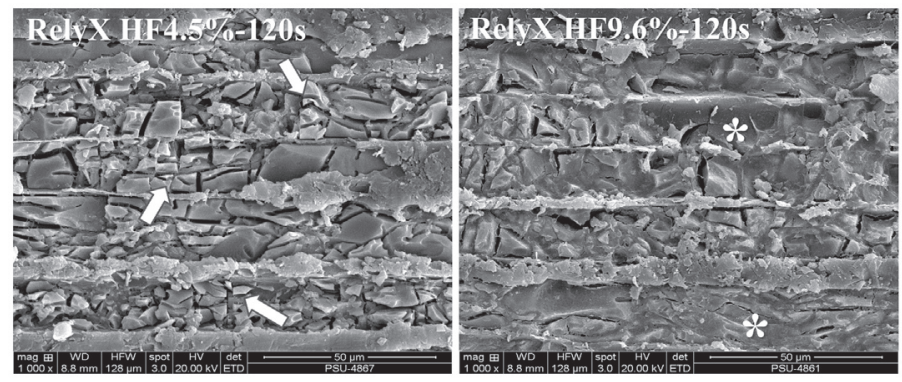

Fig. 2 High magnification (1,000×) SEM micrograph of RelyX Fiber Posts treated with HF4.5\%-120s and HF9.6\%-120s. The glass fibers were etched into small pieces (arrows) when treated with HF at $4.5 \%$ for $120 \mathrm{~s}$, while the etched fibers were dislodged from the surface leaving fiber imprint lines on the zirconia-filled matrix (asterisks), after treatment with $\mathrm{HF}$ at $9.6 \%$ for $120 \mathrm{~s}$.

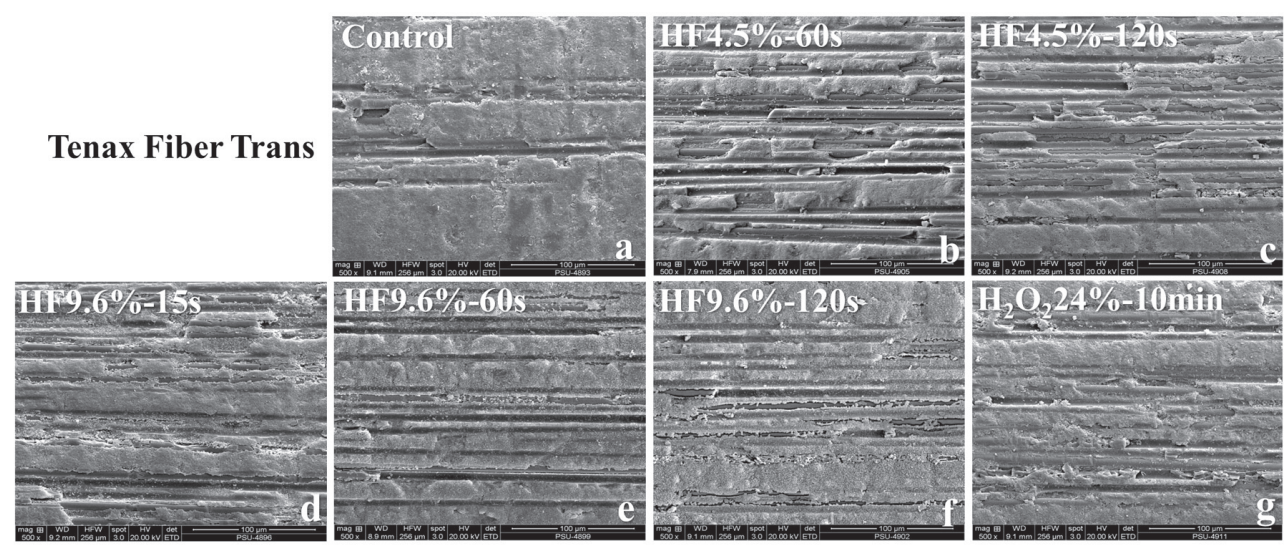

Fig. 3 SEM micrographs of Tenax Fiber Trans surfaces before and after various surface treatments.

In the control group, the post surface was tightly covered by the resin matrix with only few fibers exposed on the outer surface (a). The surface appeared intact when treated with HF9.6\%-15s or $\mathrm{H}_{2} \mathrm{O}_{2} 24 \%-10$ min (a and g). Some glass fiber bundles were removed when the post was treated with HF4.5\%- 60s (b). Glass fibers on the outer surface of a post were mostly wiped out when the post was immersed in $\mathrm{HF}$ at $4.5 \%$ for 120 s, or in $\mathrm{HF}$ at $9.6 \%$ for 60 or 120 $\mathrm{s}(\mathrm{c}, \mathrm{e}$, and f). 

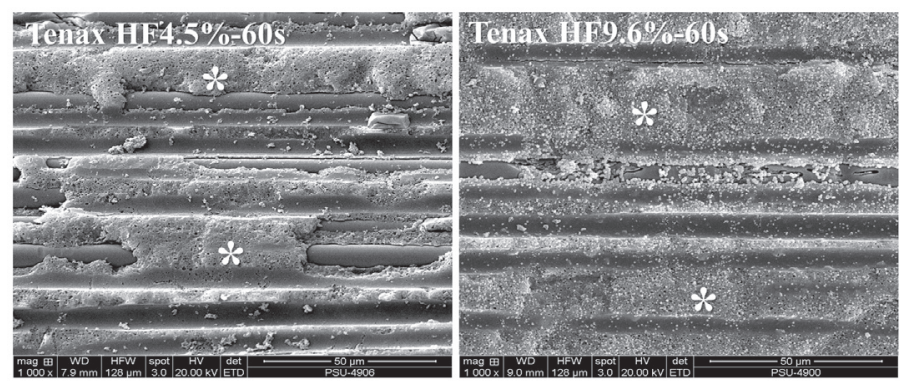

Fig. 4 High magnification (1,000×) SEM micrograph of Tenax Fiber Trans posts treated with HF at $4.5 \%$ and $9.6 \%$ for $60 \mathrm{~s}$. Several micro-pores inside the resin matrix are observed (asterisks).

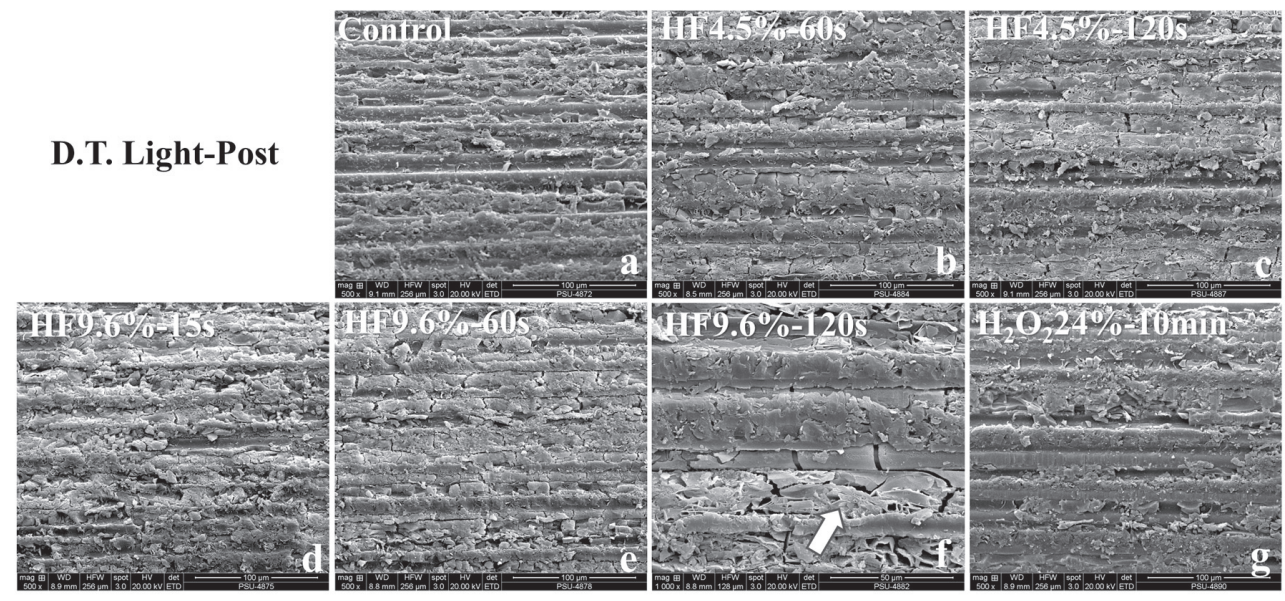

Fig. 5 SEM micrographs of D.T. Light-Post surfaces.

In the control group, the post surface was covered by the resin matrix with some fibers exposed on the outer surface (a). The surfaces treated with $\mathrm{H}_{2} \mathrm{O}_{2} 24 \%$-10min seem unchanged compared to control (g). The post surfaces treated with $\mathrm{HF}$ at $4.5 \%$ for $60 \mathrm{~s}$ or $120 \mathrm{~s}$, as well as with $\mathrm{HF}$ at $9.6 \% 15 \mathrm{~s}$ or $60 \mathrm{~s}$, show many crack lines in the fibers (b-e). In the group treated strong acid for a long time, HF9.6\%-120s, several pores and cracks have appeared on the post surface (f-arrow).

$\mathrm{H}_{2} \mathrm{O}_{2} 24 \%$-10min seemed unchanged compared to control (Fig. 5g). The post surfaces treated with $\mathrm{HF}$ at $4.5 \%$ for $60 \mathrm{~s}$ or $120 \mathrm{~s}$, as well as with $\mathrm{HF}$ at $9.6 \% 15 \mathrm{~s}$ or $60 \mathrm{~s}$, showed many crack lines in the fibers (Figs. 5b-e). In the group treated strong acid for a long time, HF9.6\%120 s, several pores and cracks have appeared on the post surface as shown by the arrow in Fig. 5 f.

\section{DISCUSSION}

The maximum loads in a three-point bending test were determined experimentally, for each type of fiber post with each alternative surface treatment. The flexural strength calculated from the ultimate failure load and the supported span does not appropriately represent the failure of a fiber-reinforced material, because the loading force always drops when some fibers break while the outer surface still appears intact. As some fibers were broken, the loading force would suddenly drop, as shown in Fig. 6. This could be the initiation of the fiber post failure. Clinically, the broken fibers or dislodgement of fibers from resin matrix could initially relate to displacement of the restoration, and consequent failure. Several previous studies have therefore used the maximum load or $\mathrm{F}_{\max }$ to calculate flexural strengths of fiber posts ${ }^{17-19)}$. Navais et al. reported that flexural strength test of the fiber post was valid as long as the taper portion of the post was limited in length ${ }^{20)}$. There were 3 types of fiber post in our present study, differing in geometry and size, including length of the taper portion. The maximum loads are here displayed to allow comparisons between the alternative surface treatments, not for comparisons between the types of tested posts. The modulus of elasticity was not calculated and reported in the present study because the taper of the post shape, which was not appropriate to use the surface area for calculation as well. Load-deflection ratios were therefore described to indirectly represent 


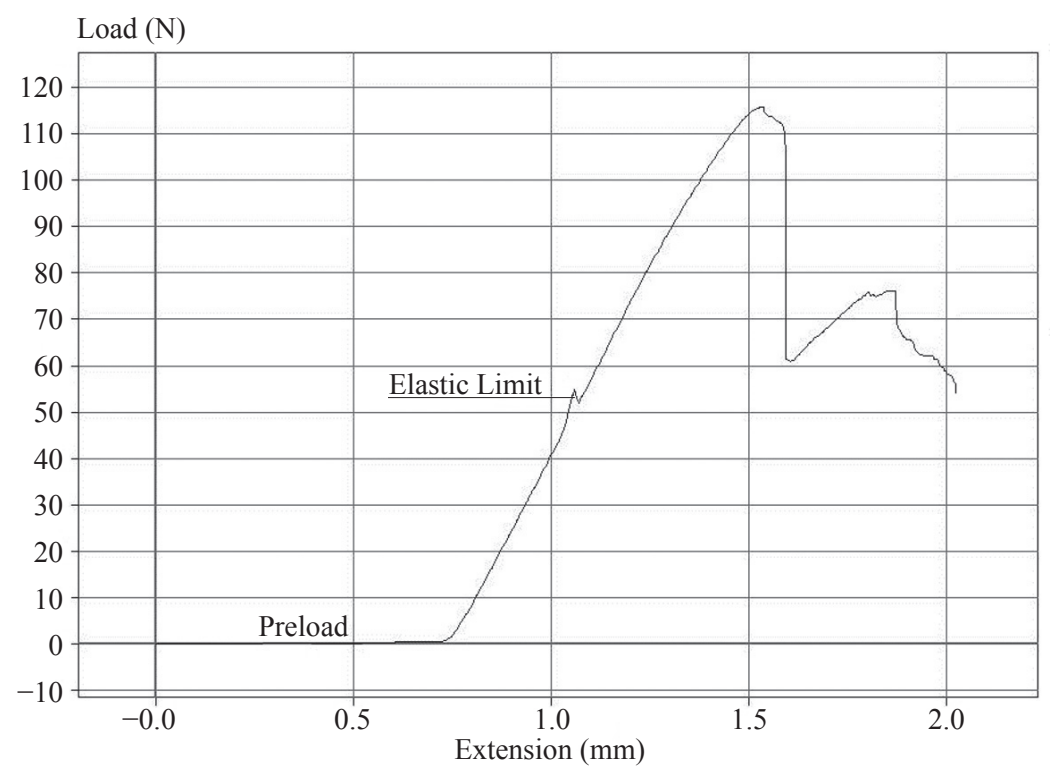

Fig. 6 This load vs. extension graph demonstrates the typical failure pattern of a fiber post under three-point flexural loading.

There is a tapered drop in the load when fibers start to break, followed by the actual failure and a sharp deep drop.

the elastic property of the test posts and to compare them among different surface treatments within the same type of post.

The results reveal that when etching with $\mathrm{HF}$, the concentrations 4.5 and $9.6 \%$ did not affect strength of the fiber post, regardless its type. HF has been used for etching glass-ceramic composites due to its ability to dissolve silica-based substrates, in order to provide micromechanical retention at the interfacial surface. In addition, etching can clean by removing oxides and impurities from some metal surfaces without dissolving. $\mathrm{HF}$ is classified as a weak acid, although it has strong ability to etch glass surfaces ${ }^{21)}$. Etching effects might only impact a thin outer surface layer, which is adequate to improve the mechanical retention without loss of internal material strength. The topography of etching patterns, as seen in the SEM pictures, showed plenty of cracked fibers, possibly causing some apprehension in clinicians regarding the strength of a fiber post after surface etching. It would be reasonable to expect that such etching pattern damage could weaken the post. However, the present result has clarified that the no strength loss accompanied the observed patterns.

Correspondingly with the strength data, loaddeflection ratios of the posts were not affected by surface treatments. It can be noted that the deflection of the post responding to the applied load, before fibers start breaking (taper drop in the graph), would not change after surface treatments. The alteration at outer surfaces might be negligible to affect the elastic property of the posts. RelyX Fiber Post seemed to have higher rigidity than those of other posts. This might be due to the matrix of this fiber post, which contains epoxy resin and zirconia fillers. The control group of RelyX Fiber Post seemed to have a higher load-deflection ratio than those of the surface treated groups although the significant difference was not detected. The non-homogeneous of the resin matrix could create interfaces between resin and fillers, and allowed water penetration during the surface treatment procedures. The rigidity of the post might be slightly declined due to this reason, compared to the homogeneous matrix of Tenax fiber Trans and D.T. Light-Post.

Etching time and $\mathrm{HF}$ concentration were found to have negligible effects on the fiber post strength and elastic property, although the severity of etching patterns differed remarkably between the alternative treatments. For RelyX Fiber Post, the long 120 s etching time strongly etched the glass fibers on the post surfaces, and broken pieces of glass fiber could be observed with both HF concentrations as shown with the arrows in Fig. 2. The SEM micrograph of an untreated RelyX Fiber Post showed that some fibers were exposed on the surface, partially covered with resin matrix, and $\mathrm{HF}$ treatment specifically etched the inorganic glass fibers. Manifest etching patterns were therefore observed. The Tenax Fiber Trans had the fibers mostly sheltered by a dense resin matrix, with only few fibers exposed. In addition, the fiber-matrix interfaces of Tenax Fiber Trans might not have strong adhesion: the etched fibers tended to dislodge from the surface rather than leaving broken fiber pieces behind. The SEM micrograph displayed imprints of fibers on the surfaces (Fig. 3). On D.T. Light-Posts, many glass fibers could be observed on the surface of an untreated post. One could expect then that this type of post would be easily etched to have 


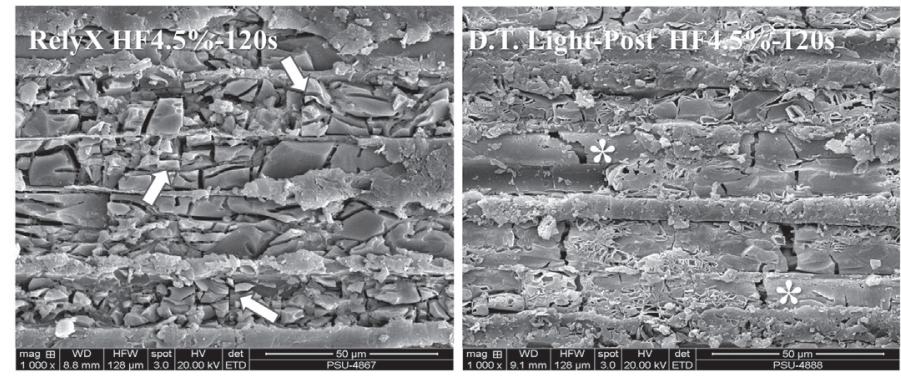

Fig. 7 High magnification (1,000×) of SEM micrographs of RelyX Fiber Post and D.T. Light-Post surfaces treated with HF at $120 \%$ for $120 \mathrm{~s}$.

The glass fibers of RelyX Fiber Post were etched into small pieces (arrows), whereas the quartz fibers of D.T. LightPost revealed only some crack lines (asterisks).

clear etching patterns. However, the etched surfaces of D.T. Light-Post were not remarkable in the same way as those of RelyX Fiber Post (Fig. 7). In the HF4.5\%-120s group, the glass fibers of RelyX Fiber Post were etched into small pieces (Fig. 7-arrows), whereas the quartz fibers of D.T. Light-Post revealed only some crack lines (Fig. 7-asterisks). This might be due to different types of fiber in these two posts, as the RelyX Fiber Post contains glass fibers while D.T. Light-Post contains quartz fibers. The latter are stronger and stiffer than glass fibers. Therefore, the fibers in D.T. Light-Post were more resistant to $\mathrm{HF}$ etching than those in RelyX Fiber Post. However, the resistance to acid etching of the fibers did not refer to the strength of the posts. Average maximum load from three point-flexural testing of RelyX Fiber Post was considerably higher than that of D.T. LightPost. The strength of fiber posts could be affected by various factors such as post geometry, post size, type of resin matrix, or bonding between fibers and matrix.

It was found that treating the post surfaces by immersion in $24 \% \mathrm{H}_{2} \mathrm{O}_{2}$ for $10 \mathrm{~min}$ did not alter the maximum load of Tenax Fiber Trans or D.T. LightPost, by comparison to untreated controls. However, with the RelyX Fiber Post, the maximum load for the $\mathrm{H}_{2} \mathrm{O}_{2}$ 24\%-10min group differed from those of HF9.6\%60s and HF9.6\%-120s treatment groups, at a mildly significant level. The average maximum load for the $\mathrm{H}_{2} \mathrm{O}_{2} 24 \%-10 \mathrm{~min}$ group was lower than those of the other treatment groups, although without statistical significance. The $\mathrm{H}_{2} \mathrm{O}_{2}$ has been promoted for use in treating fiber post surfaces, because some clinicians are concerned about the effects of $\mathrm{HF}$ on the integrity of fiber posts ${ }^{8)}$. Additionally, the preparation of $\mathrm{H}_{2} \mathrm{O}_{2}$ is more convenient because this agent is widely used in dental treatments, for example as a root canal irrigating agent. It was presumed that $\mathrm{H}_{2} \mathrm{O}_{2}$ could dissolve the resin on the fiber surfaces and create more micromechanical retention without damaging the fibers ${ }^{22)}$. In the SEM micrographs of the current study, it was found that the surface topographies of all the tested fibers post types were very similar to the untreated posts. With the exception of RelyX Fiber Post, the strengths were unaffected by hydrogen peroxide treatment. The matrix in the RelyX Fiber Post is the composite material containing epoxy resin with zirconia filler, which might be the reason for loss of strength after immersion in $\mathrm{H}_{2} \mathrm{O}_{2}$. It has been reported that the hardness of a composite resin with zirconia filler significantly decreases after immersion in $40 \% \mathrm{H}_{2} \mathrm{O}_{2}$ bleaching agent, and the hydrogen peroxide could diffuse and disturb the resin-filler interfaces causing micro-cracks, depending on the materials ${ }^{23)}$. This would subsequently impair the strength of the post, as observed in the present experiment. The others types of post in the current study had homogeneous resin without filler. While statistically significant differences in strength were not found in the current study, the observations do encourage further studies on how $\mathrm{H}_{2} \mathrm{O}_{2}$ treatment affects the properties of fiber posts.

The results of this study indicate that fiber post surface pretreatments with hydrofluoric acid and hydrogen peroxide are acceptable practices without negative impact on post strength. Clinicians can utilize these treatment agents to improve the bond strength between the fiber post and the luting medium, without deterioration of the posts.

\section{CONCLUSION}

Fiber post surface etching using hydrofluoric acid and hydrogen peroxide with various alternative protocols were studied, and no adverse effects on the flexural properties were found, for three tested types of commercially available fiber post. However, the SEM imaging of surface topography showed differences between the protocols tested.

\section{ACKNOWLEDGMENT}

The authors thank Seppo Karrila and Research and Development Office for improving the use of English in the manuscript. 


\section{REFERENCES}

1) Aggarwal V, Singla M, Miglani S, Kohli S. Comparative evaluation of fracture resistance of structurally compromised canals restored with different dowel methods. J Prosthodont 2012; 21: 312-316.

2) Akkayan B, Gulmez T. Resistance to fracture of endodontically treated teeth restored with different post systems. J Prosthet Dent 2002; 87: 431-437.

3) Hayashi M, Sugeta A, Takahashi Y, Imazato S, Ebisu S. Static and fatigue fracture resistances of pulpless teeth restored with post-cores. Dent Mater 2008; 24: 1178-1186.

4) Ferrari M, Cagidiaco MC, Goracci C, Vichi A, Mason PN, Radovic I,Tay F. Long-term retrospective study of the clinical performance of fiber posts. Am J Dent 2007; 20: 287-291.

5) Parisi C, Valandro LF, Ciocca L, Gatto MRA, Baldissara P. Clinical outcomes and success rates of quartz fiber post restorations: A retrospective study. J Prosthet Dent 2015; 114: $367-372$.

6) Aksornmuang J, Nakajima M, Foxton RM, Panyayong W, Tagami J. Regional bond strengths and failure analysis of fiber posts bonded to root canal dentin. Oper Dent 2008; 33: 636-643.

7) Soares CJ, Santana FR, Pereira JC, Araujo TS, Menezes MS. Influence of airborne-particle abrasion on mechanical properties and bond strength of carbon/epoxy and glass/bisGMA fiber-reinforced resin posts. J Prosthet Dent 2008; 99: 444-454.

8) Monticelli F, Toledano M, Tay FR, Sadek FT, Goracci C, Ferrari M. A simple etching technique for improving the retention of fiber posts to resin composites. J Endod 2006; 32: 44-47.

9) Vano M, Goracci C, Monticelli F, Tognini F, Gabriele M, Tay FR, Ferrari M. The adhesion between fibre posts and composite resin cores: the evaluation of microtensile bond strength following various surface chemical treatments to posts. Int Endod J 2006; 39: 31-39.

10) D'Arcangelo C, D'Amario M, Prosperi GD, Cinelli M, Giannoni M, Caputi S. Effect of surface treatments on tensile bond strength and on morphology of quartz-fiber posts. J Endod 2007; 33: 264-267.

11) Guler AU, Kurt M, Duran I, Uludamar A, Inan O. Effects of different acids and etching times on the bond strength of glass fiber-reinforced composite root canal posts to composite core material. Quintessence Int 2012; 43: e1-8.

12) Naves LZ, Santana FR, Castro CG, Valdivia AD, Da Mota AS, Estrela C, Correr-Sobrinho L, Soares CJ. Surface treatment of glass fiber and carbon fiber posts: SEM characterization. Microsc Res Tech 2011; 74: 1088-1092.

13) Schmage P, Cakir FY, Nergiz I, Pfeiffer P. Effect of surface conditioning on the retentive bond strengths of fiberreinforced composite posts. J Prosthet Dent 2009; 102: 368-377.

14) Samimi P, Mortazavi V, Salamat F. Effects of heat treating silane and different etching techniques on glass fiber post push-out bond strength. Oper Dent 2014; 39: E217-224.

15) Valandro LF, Yoshiga $\mathrm{S}$, de Melo RM, Galhano GAP, Mallmann A, Marinho CP, Bottino MA. Microtensile bond strength between a quartz fiber post and a resin cement: Effect of post surface conditioning. J Adhes Dent 2006; 8: 105111.

16) de Sousa Menezes M, Queiroz EC, Soares PV, Faria-e-Silva AL, Soares CJ, Martins LR. Fiber post etching with hydrogen peroxide: effect of concentration and application time. $\mathrm{J}$ Endod 2011; 37: 398-402.

17) Cheleux N, Sharrock PJ. Mechanical properties of glass fiberreinforced endodontic posts. Acta Biomater 2009; 5: 32243230 .

18) Stewardson DA, Shortall AC, Marquis PM, Lumley PJ. The flexural properties of endodontic post materials. Dent Mater 2010; 26: 730-736.

19) Zicari F, Coutinho E, Scotti R, Van Meerbeek B, Naert I. Mechanical properties and micro-morphology of fiber posts. Dent Mater 2013; 29: e45-e52.

20) Novais VR, Versluis A, Correr-Sobrinho L, Soares CJ. Threepoint bending testing of fibre posts: critical analysis by finite element analysis. Int Endod J 2011; 44: 519-524.

21) Pauling L. Why is hydrofluoric acid a weak acid? An answer based on a correlation of free energies, with electronegativities. J Chem Educ 1956; 33: 16.

22) Yenisey M, Kulunk S. Effects of chemical surface treatments of quartz and glass fiber posts on the retention of a composite resin. J Prosthet Dent 2008; 99: 38-45.

23) Alqahtani MQ. Tooth-bleaching procedures and their controversial effects: A literature review. Saudi Dent J 2014; 26: $33-46$. 